\title{
Fertilization failure is not associated with sperm motility following human in vitro fertilization
}

\begin{abstract}
In reproductive medicine, total and partial fertilization failure (TFF/PFF) following conventional in vitro fertilization (cIVF) has frustrated patients. To address this issue of the role of sperm motility in TFF/PFF, we have conducted a systematic analysis of the sperm parameters that affect TFF/PFF following human cIVF. We show here that the sperm motility becomes more vigorous in TFF/PFF patients, and sperm hyper activation induced by progesterone is unlikely to induce TFF/PFF. However, we found that the lower number of oocytes retrieved and the reduction in sperm binding to the zona pellucida observed among TFF/PFF patients might induce fertilization failure. We strongly believe that sperm motility is not sufficient to induce TFF/PFF, whereas the interaction between sperm and oocyte might help enhance fertilization and prevent fertilization failure during human IVF
\end{abstract}

Keywords: fertilization failure, sperm motility, hyperactivation, sperm zona binidng

\author{
Volume I Issue I - 2014 \\ Yoku Kato,' Yukiko Asano,' Maki Tsunekawa,' \\ Saori Sato,' Masashi Shimizu, ${ }^{2}$ Yasutaka \\ Murata, ${ }^{2}$ Hisanori Oguri' \\ 'Department of infertility, Royal Bell Clinic, Japan \\ ${ }^{2}$ Department of infertility, Angel Bell Hospital, Japan
}

Correspondence: Yoku Kato, Department of Infertility, Royal Bell Clinic, Narumi-cho Mizuhiroge 93-195, Midori-ku, Nagoya, Aichi, 458-080I, Japan, Tel +8I-052-879-6673,

Email yoku-kato@kishokai.or.jp

Received: August 29, 2014 | Published: September 12, 2014
Abbreviations: TFF, total fertilization failure; PFF, partial fertilization failure; cIVF, conventional in vitro fertilization; CASA, computer-assisted sperm analysis; ZP, zona pellucida; IVF, in vitro fertilization

\section{Introduction}

In reproductive medicine, total and partial fertilization failure (TFF/PFF) following conventional in vitro fertilization (cIVF) has frustrated patients, adding emotional pain and financial burden to painful treatment. The incidence of TFF/PFF following cIVF, in the presence of sperm with progressive motility, has been reported to be $4-16 \% .{ }^{1}$ To address this issue of the role of sperm motility in $\mathrm{TFF} / \mathrm{PFF}$, we have conducted a systematic analysis of the sperm parameters that affect TFF/PFF following human cIVF. These include semen parameters, sperm motility, the sperm's ability to bind to the zona pellucida (ZP), fertilization parameters, and the progesterone concentration in the insemination media used.

\section{Materials and methods}

This retrospective study was approved by our Institutional Ethical Board on 21th, August 2014. This study evaluated 613 cycles from patients who had been treated at the Department of Infertility, Angel Bell Hospital between May 2010 and December 2013, and at the Royal Bell Clinic between April and December 2013. Sperm were obtained from the male partner following masturbation, and were processed using the discontinuous gradient and swim-up technique. Three hours after oocyte retrieval, oocytes were fertilized using cIVF. Five hours after insemination, denuded oocytes without a second polar body were considered to be unfertilized. ${ }^{2}$ After $18-20$ hours insemination, fertilization was determined by checking for the presence of two pronuclei and two polar body. Sperm analysis was performed using computer-assisted sperm analysis (CASA), according to published guidelines.${ }^{3,4}$ For statistical analyses, differences were evaluated using the t-test, and considered significant at $\mathrm{P}<0.05$.

\section{Results}

The CASA parameters for patients with and without TFF/PFF are listed in Table 1. Among patients with TFF/PFF, most CASA parameters had increased by the time the sperm were suspended following swim-up $(\mathrm{P}<0.05)$, with the exception of linearity and amplitude of lateral head displacement. As well, we observed a significant increase in all CASA parameters for patients without TFF/PFF $(\mathrm{P}<0.05)$. The linearity of sperm motility was steady at approximately 0.4 among TFF/PFF patients. As well, the amplitude of lateral head displacement exhibited a constant trend at approximately 12.0 Hz. Sperm hyperactivation following swim-up was detected in $68.4 \%$ and $78.9 \%$ of patients with and without TFF/PFF, respectively. There was no significant difference in the average progesterone concentration for the insemination media used to treat patients with and without TFF/PFF (119 and $100 \mathrm{ng} / \mathrm{mL}$ ). A significant difference was detected in the number of oocytes retrieved for patients with and without TFF/PFF (5.6 vs. 8.7 oocytes; $\mathrm{P}<0.01$ ). The prevalence of two pronuclei among TFF/PFF patients was significantly lower than that among patients without TFF/PFF $(1.9 \%$ vs. $73.6 \%$; $\mathrm{P}<0.0001)$. For TFF/PFF patients, the number of sperm bound to the ZP was substantially lower than that for patients without TFF/PFF.

\section{Discussion}

Regarding sperm motility, the significant increase in TFF/ PFF patients' sperm parameters with the exception of linearity and amplitude of lateral head displacement after swim-up indicates increase in vigor, whereas the non-TFF/PFF patients' parameters also increased significantly. Moreover, we found that sperm hyperactivation in most patients was induced by progesterone, and the progesterone concentrations were similar for both groups of patients. Progesterone is present throughout the female genital tract, reaching a peak concentration in the cumulus matrix that surrounds the oocyte. Progesterone is also known to stimulate several sperm functions, including hyperactivation ${ }^{5}$ and acrosome reaction. ${ }^{6}$ Hyperactivated motility might assist the sperm in penetrating the $\mathrm{ZP}$, which is a critical process for fertilization. ${ }^{7}$ Thus, we conclude that sperm motility becomes more vigorous in TFF/PFF patients, and sperm hyperactivation induced by progesterone is unlikely to induce TFF/PFF. 
Table I Semen and sperm parameters for patients with total and partial fertilization failure (TFF and PFF)

\begin{tabular}{|c|c|c|c|}
\hline & Patients & & $P$ value \\
\hline & Without TFF/PFF $(n=580) \#$ & With TFF/PFF $(n=33) \#$ & \\
\hline Maternal age & $35.7 \pm 0.2$ & $36.6 \pm 0.9$ & NS \\
\hline Paternal age & $36.6 \pm 0.2$ & $37.2 \pm 1.1$ & NS \\
\hline No. of oocyte retrieved & $8.7 \pm 0.3$ & $5.6 \pm 0.9$ & $\mathrm{P}<0.0 \mathrm{I}$ \\
\hline Total fertilization (\%) & $91.2 \pm 0.7$ & $6.8 \pm 2.5$ & $P<0.000$ I \\
\hline Two pronuclei (\%) & $73.6 \pm I . I$ & $1.9 \pm 1.0$ & $\mathrm{P}<0.000 \mathrm{I}$ \\
\hline Semen volume $(\mathrm{ml})$ & $3.0 \pm 0.1$ & $3.0 \pm 0.3$ & NS \\
\hline Sperm concentration $(\times 106 / \mathrm{ml})$ in semen & $67.1 \pm 2.4 *$ & $52.6 \pm 6.5^{*}$ & NS \\
\hline Sperm concentration $(\times 106 / \mathrm{ml})$ after swim-up & II $.0 \pm 0.4$ & $8.3 \pm 6.5$ & NS \\
\hline Motility (\%) in semen & $45.5 \pm 0.1 *$ & $40.4 \pm 0.3 *$ & NS \\
\hline Motility (\%) after swim-up & $98.7 \pm 0.2$ & $93.9 \pm 2.7$ & NS \\
\hline Straight-line velocity $(\mu \mathrm{m} / \mathrm{s})$ in semen & $20.2 \pm 0.4 *$ & $22.1 \pm 2.4^{*}$ & NS \\
\hline Straight-line velocity $(\mu \mathrm{m} / \mathrm{s})$ after swim-up & $40.5 \pm 0.8$ & $42.1 \pm 3.0$ & NS \\
\hline Curvilinear velocity $(\mu \mathrm{m} / \mathrm{s})$ in semen & $47.5 \pm 1.0 *$ & $50.0 \pm 4.6^{*}$ & NS \\
\hline Curvilinear velocity $(\mu \mathrm{m} / \mathrm{s})$ after swim-up & $119.8 \pm 1.6$ & $1 \mid 4.7 \pm 6.0$ & NS \\
\hline Linearity in semen & $0.4 \pm 0.01 *$ & $0.4 \pm 0.03$ & NS \\
\hline Linearity after swim-up & $0.36 \pm 0.01$ & $0.4 \pm 0.03$ & NS \\
\hline Beat cross frequency $(\mu \mathrm{m})$ in semen & $1.2 \pm 0.03 *$ & $1.2 \pm 0.1 *$ & NS \\
\hline Beat cross frequency $(\mu \mathrm{m})$ after swim-up & $2.7 \pm 0.04$ & $2.6 \pm 0.1$ & NS \\
\hline Amplitude of lateral head displacement $(\mathrm{Hz})$ in semen & $12.0 \pm 0.1 *$ & $12.0 \pm 0.4$ & NS \\
\hline Amplitude of lateral head displacement $(\mathrm{Hz})$ after swim-up & $13.0 \pm 0.2$ & $12.4 \pm 0.6$ & NS \\
\hline Hyperactivation (\%) in semen $\%$ & $0.50 \% *$ & $0 \% *$ & NS \\
\hline Hyperactivation (\%) after swim-up $\%$ & $78.90 \%$ & $68.40 \%$ & NS \\
\hline No. of sperm binding zona pellucida (No. of sperm/oocyte) & $3.5 \pm 0.4(n=55)$ & $1.5 \pm 0.4(n=34)$ & $P<0.05$ \\
\hline Progesterone concentration in insemination media & $100.0 \pm 16.0(n=28)$ & $1 \mid 8.5 \pm 13.6(n=4)$ & NS \\
\hline
\end{tabular}

$*_{n}=$ number of patients. The number of patients assessed by CASA: 220 patients without TFF/PFF and 19 patients with TFF/PFF.

*Significant differences between semen before swim-up and sperm suspension after swim-up ( $\mathrm{t}$-test $\mathrm{P}<0.05)$.

※The number of patients with induced sperm hyperactivation divided by the total number of patients. Induction of hyperactivation in patients was assessed using standard criteria to identify hyperactivation, $\geq 100 \mu \mathrm{m} / \mathrm{s}$ average curvilinear velocity, $\leq 0.5$ average linearity, $\geq 8 \mathrm{~Hz}$ average amplitude of lateral head displacement.

Interestingly, we found the reduction in sperm binding to the $\mathrm{ZP}$, oocyte retrieval, and fertilization observed among patients with TFF/PFF. Recently, it has been reported that the O-glycan of ZP3 can act as a sperm head receptor. ${ }^{8,9}$ As well, the interaction between the Izumo protein on the sperm membrane ${ }^{10}$ and the Juno receptor on the oolemma ${ }^{11}$ are required for successful fertilization. Therefore, we suggest that the interactions between sperm and oocyte should be considered to promote fertilization and prevent TFF or PFF.

We strongly believe that sperm motility is not sufficient to induce $\mathrm{TFF} / \mathrm{PFF}$, whereas the interaction between sperm and oocyte might help enhance fertilization and prevent fertilization failure during IVF.

\section{Acknowledgments}

None.

\section{Conflicts of interest}

Author has no any conflict of interest to declare.

\section{References}

1. Beck-Fruchter R, Lavee M, Weiss A, et al. Rescue intracytoplasmic sperm injection: a systematic review. Fertil Steril. 2014;101(3):690-698.

2. Shimizu M, Nagashima Y, Tomoto K, et al. Examination of the proper criteria for rescue intracytoplasmic sperm injection. $J$ Fertil Implant. 2006;29(1):91-95.

3. World Health Organization (WHO). Department of reproductive health and research. 5th edn. WHO laboratory manual for the examination and processing of human semen, Switzerland; 2010.287 p.

4. Hamdi SM, Vieitez G, Jaspard B, et al. Effects of human follicular fluid and high-density lipoproteins on early spermatozoa hyperactivation and cholesterol efflux. J Lipid Res. 2010;51(6):1363-1369. 
5. Lishko PV, Botchkina IL, Kirichok Y. Progesterone activates the principal $\mathrm{Ca}^{+}$channel of human sperm. Nature. 2011;471(7338):387-391.

6. Sanchez-Cardenas C, Servin Vences MR, Jose O, et al. Acrosome reaction and $\mathrm{Ca} 2^{+}$imaging in single human spermatozoa: new regulatory roles of [Ca2 $\left.{ }^{+}\right]$i. Biol Reprod (in press). 2014.

7. Suarez SS. Control of hyperactivation in sperm. Hum Reprod Update. 2008;14(6):647-657.

8. Visconti PE, Florman HM. Mechanisms of sperm-egg interactions: between sugars and broken bonds. Sci Signal. 2010;3(142):pe35.
9. Han L, Monne M, Okumura H, et al. Insights into egg coat assembly and egg-sperm interaction from the X-ray structure of full-length ZP3. Cell. 2010;143(3):404-415.

10. Inoue $\mathrm{N}$, Ikawa $\mathrm{M}$, Isotani $\mathrm{A}$, et al. The immunoglobulin superfamily protein Izumo is required for sperm to fuse with eggs. Nature. 2005;434(7030):234-238.

11. Bianchi E, Doe B, Goulding D, et al. Juno is the egg Izumo receptor and is essential for mammalian fertilization. Nature. 2014;508(7497):483487. 